\title{
ESTADO NUTRICIONAL DE CRIANÇAS E ADOLESCENTES COM SÍNDROME DE DOWN: REVISÃO INTEGRATIVE
}

\section{REVISÃO INTEGRATIVA}

SONG, Berty Ru Ping ${ }^{1}$

MANFREDI, Paula ${ }^{2}$

SOUZA, Isabel Fernandes de ${ }^{3}$

SONG, Berty Ru Ping. MANFREDI, Paula. SOUZA, Isabel Fernandes de. Estado nutricional de crianças e adolescentes com Síndrome de Down: Revisão integrativa. Revista Científica Multidisciplinar Núcleo do Conhecimento. Ano 05, Ed. 11, Vol. 19, pp. 55-70. Novembro de 2020. ISSN: 2448-0959, Link de acesso: https://www.nucleodoconhecimento.com.br/nutricao/estado-nutricional

\section{RESUMO}

Introdução: A obesidade é uma doença crônica definida como acúmulo anormal ou excessivo de gordura corporal devido ao balanço energético positivo. Porém, a epidemia é pouco estudada em pessoas com Síndrome de Down (SD). A população com SD é mais suscetível a desenvolver sobrepeso e obesidade, atingindo de um terço a metade da população. Objetivo: Evidenciar a prevalência de sobrepeso e obesidade em crianças e adolescentes com Síndrome de Down em um decênio (2009-

${ }^{1}$ Graduanda em Nutrição no Centro Universitário União das Américas. Estagiária do Centro Especializado de Reabilitação IV (CER IV).

2 Orientadora. Graduação em nutrição. Mestre em Envelhecimento Humano pela Universidade de Passo Fundo. Docente do centro universitário União das Américas Foz do Iguaçu.

${ }^{3}$ Doutora em ciências da Engenharia da Produção. Docente do Curso de Fisioterapia. Orientadora em Iniciação Científica. Centro Universitário União das Américas. 
2019). Metodologia e resultados: A busca dos artigos se deu no período de 03 a 28 de agosto de 2020, consultando as bases de dados PubMed, Scielo e LILACS. Dos 473 documentos recuperados, 13 estudos foram incluídos na revisão. Considerações Finais: Com base nos dados levantados neste estudo, foi possível identificar uma prevalência alarmante de sobrepeso e obesidade em crianças e adolescentes com SD. Tal quadro acomete indivíduos com SD saudáveis e com comorbidades, de ambos os sexos independendo da idade e o grau de deficiência.

Palavras-chave: Síndrome de Down, obesidade, estado nutricional.

\section{INTRODUÇÃO}

A obesidade é uma doença crônica que, segundo a Organização Mundial da Saúde, pode ser definida como acúmulo anormal ou excessivo de gordura corporal devido ao balanço energético positivo (WHO, 1998). Ela pode ser classificada a partir do Índice de Massa Corporal (IMC), percentual de gordura corporal (\%GC), relação cinturaquadril e bioimpedância elétrica (BIA) (WRIGHT et al., 2008).

Nas últimas décadas esse quadro vem aumentando de maneira alarmante, comprometendo a saúde e o bem-estar dos indivíduos e, por esse motivo a obesidade tornou-se uma das maiores preocupações de saúde pública¹. Porém, a epidemia é pouco estudada em populações específicas, como por exemplo, pessoas com Síndrome de Down (THEODORO; BLASCOVI-ASSIS, 2009).

A Síndrome de Down (SD) é uma condição genética em que os indivíduos apresentam um cromossomo a mais no par 21, por isso é conhecida também como trissomia do cromossomo 21. É considerada a anormalidade mais frequente nos humanos afetando 1 a cada 700-1000 nascimentos (CHAVE; CAMPOS; NAVARRO, 2012).

Estudos apontam que crianças com SD são mais suscetíveis a desenvolver sobrepeso e obesidade, atingindo de um terço a metade da população. Tal quadro pode acarretar outros problemas de saúde concomitantes como doenças cardiovasculares, dislipidemias, hipertensão arterial sistêmica (HAS), síndrome da apneia obstrutiva do 
sono (SAOS), entre outros. Além disso, crianças com sobrepeso e obesidade têm o risco aumentado de se tornarem adultos obesos (VAN GAMEREN-OOSTEROM et al., 2012; LORÍA; URRUTIA, 2009; RIMMER et al., 2009).

Crianças com SD apresentam características físicas e fisiológicas inerentes como hipotonia muscular, cardiopatia congênita, hipotireoidismo, disfunções auditivas e visuais, taxa metabólica basal diminuída e alterações gastrointestinais; além do atraso no desenvolvimento e deficiência intelectual que limita a prática de atividades físicas. Esse conjunto de complicações que acometem diretamente e/ou indiretamente os aspectos nutricionais, somado aos hábitos alimentares inadequados favorece o ganho de peso (MOURA et al., 2009; MOREIRA; EL-HANI; GUSMÃO, 2000; BULL, 2011).

Sendo assim, o presente projeto teve como objetivo evidenciar a prevalência de sobrepeso e obesidade em crianças e adolescentes com Síndrome de Down em um decênio (2009-2019), a partir de uma revisão integrativa dos estudos selecionados.

\section{MATERIAIS E MÉTODOS}

Trata-se de uma revisão integrativa da literatura, sendo esta uma das metodologias mais amplas, que consiste em realizar um levantamento bibliográfico coletando dados a partir de fontes secundárias, a fim de sintetizar os conhecimentos sobre um mesmo tópico (SOUZA; SILVA; CARVALHO, 2010). Inicialmente foi definido o tema da pesquisa e a pergunta norteadora: "Quais as evidências presentes no referencial teórico, de um decênio (2009-2019), quanto a prevalência de sobrepeso e obesidade em crianças e adolescentes com Síndrome de Down?" e logo, os descritores. A hipótese é de que, $10 \%$ apresentam obesidade e $30 \%$ apresentam sobrepeso.

Para o levantamento do material bibliográfico, a busca dos artigos se deu no período de 03 a 28 de agosto de 2020, consultando as bases de dados PubMed, Scielo e Literatura Latino-Americana e do Caribe em Ciências da Saúde (LILACS). Foram utilizados os seguintes descritores e suas combinações nas línguas portuguesa e inglesa: "Síndrome de Down", "Obesidade" e "Estado Nutricional" ("Down Syndrome", "Obesity" e "Nutritional Status"). 
Os critérios de inclusão para a seleção dos materiais foram: estudos que contemplam o problema, ou seja, sobrepeso e obesidade em crianças e adolescentes com SD; publicados em português, inglês e espanhol; publicações disponíveis na íntegra e gratuitamente que retratassem o tema abordado e publicados e indexados nos bancos de dados entre os anos 2009 e 2019. Foram excluídos artigos duplicados, que apresentaram pesquisas fora do escopo da busca ou que trataram da Síndrome de Down nos adultos e idosos, na educação física e na psicologia.

A busca nas bases de dados resultou em 473 documentos. A partir da leitura dos títulos, foi possível excluir 382 artigos que se mostraram fora do escopo da busca, sem acesso ao material na íntegra e duplicados. Logo foi feita a leitura de resumo dos artigos restantes $(n=91)$. Tal leitura possibilitou a exclusão de outros 73 artigos que não contemplavam especificamente o tema abordado (Tabela 1).

Tabela 1. Artigos excluídos após leitura de resumos

\begin{tabular}{|c|c|c|c|}
\hline Área ou tema do artigo & Descartados & Área ou tema do artigo & Descartados \\
\hline Genética & 4 & Nutrição (outros aspectos) & 7 \\
\hline Câncer & 1 & Deficiências gerais & 9 \\
\hline Hormônios & 7 & $\begin{array}{l}\text { Transtorno do Espectro } \\
\text { Autista }\end{array}$ & 2 \\
\hline Educação Física & 11 & Medicina & 3 \\
\hline Recomendações & 10 & Outros assuntos & 13 \\
\hline Família & 6 & Total descartados: & 73 \\
\hline
\end{tabular}

Fonte: Berty Ru Ping Song

Após a leitura completa dos 18 artigos restantes, 13 artigos foram integrados a revisão (Figura 1). 
Figura 1. Fluxograma do processo de seleção dos artigos pesquisados

Exclusão inicial de 382 documentos (indisponivel na íntegra, formato distinto de artigo científico ou duplicação). Exclusão de outros 72 que não contemplavam especificamente o tema abordado.

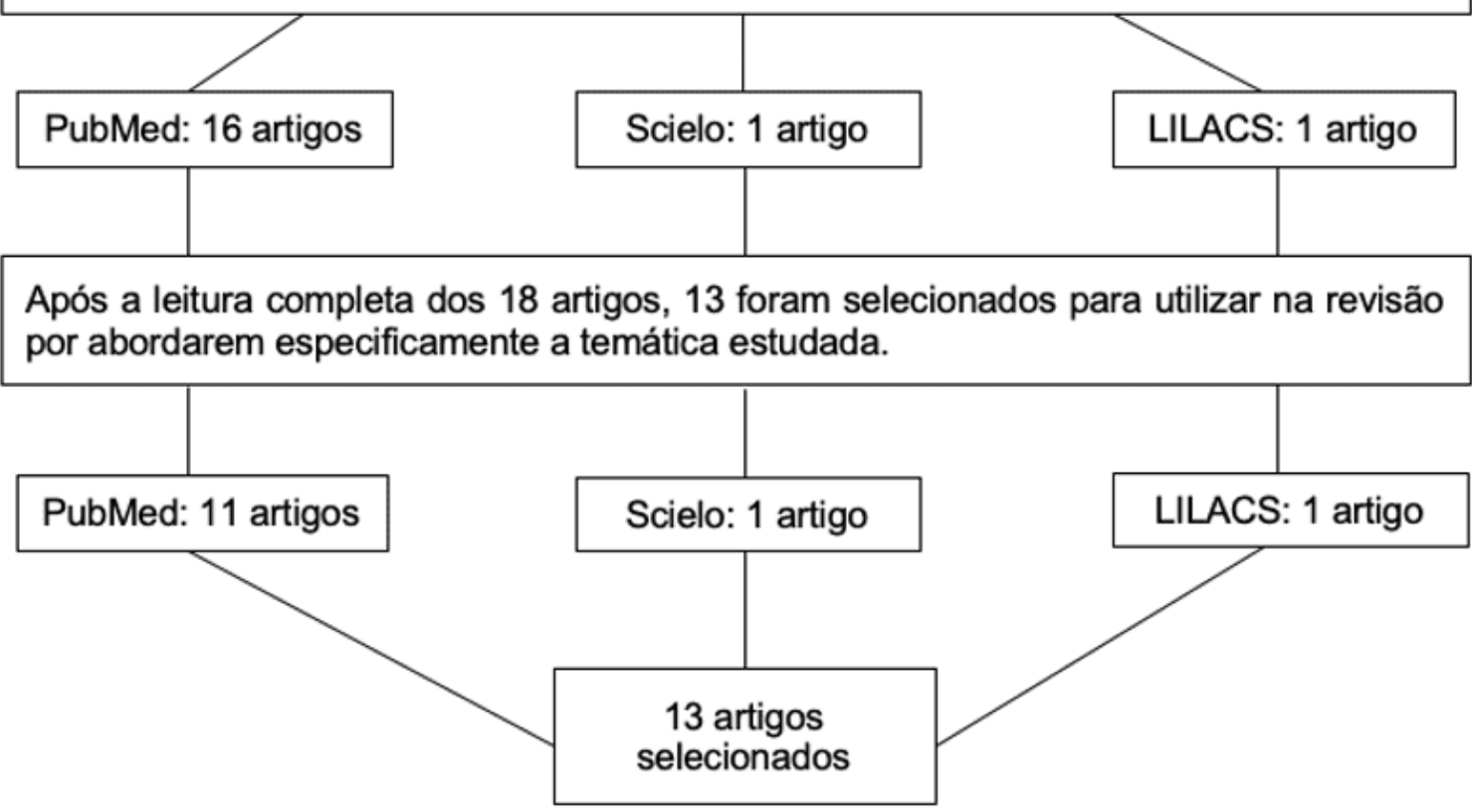

Fonte: Berty Ru Ping Song

As avaliações para a seleção dos artigos foram feitas por um único pesquisador. Dos 13 estudos que foram integrados a revisão, doze (12) se deram a partir dos descritores "Síndrome de Down" AND "obesidade" e um (1) em "Síndrome de Down" AND "estado nutricional" conforme apresentado no Quadro 1.

Quadro 1. Resultados das pesquisas

\begin{tabular}{|c|c|c|c|}
\hline Termos & $\begin{array}{l}\text { Síndrome de Down } \\
\text { AND obesidade / } \\
\text { Down Syndrome } \\
\text { AND obesity }\end{array}$ & $\begin{array}{l}\text { Síndrome } \\
\text { AND }\end{array}$ & $\begin{array}{l}\text { Síndrome de } \begin{array}{l}\text { Down } \\
\text { AND estado }\end{array} \\
\text { nutricional / Down } \\
\text { Syndrome } \\
\text { nutritional status }\end{array}$ \\
\hline $\begin{array}{l}\text { Base de } \\
\text { dados }\end{array}$ & & & \\
\hline
\end{tabular}




\begin{tabular}{|c|c|c|c|}
\hline PubMed & $\begin{array}{l}\text { Encontrados: } 214 \\
\text { Excluídos: } 204 \\
\text { Total: } 10\end{array}$ & $\begin{array}{l}\text { Encontrados: } 52 \\
\text { Excluídos: } 52 \\
\text { Total: } 0\end{array}$ & $\begin{array}{l}\text { Encontrados: } 47 \\
\text { Excluídos: } 46 \\
\text { Total: } 1\end{array}$ \\
\hline Scielo & $\begin{array}{l}\text { Encontrados: } 41 \\
\text { Excluídos: } 40 \\
\text { Total: } 1\end{array}$ & $\begin{array}{l}\text { Encontrados: } 2 \\
\text { Excluídos: } 2 \\
\text { Total: } 0\end{array}$ & $\begin{array}{l}\text { Encontrados: } 29 \\
\text { Excluídos: } 29 \\
\text { Total: } 0\end{array}$ \\
\hline LILACS & $\begin{array}{l}\text { Encontrados: } 63 \\
\text { Excluídos: } 62 \\
\text { Total: } 1\end{array}$ & $\begin{array}{l}\text { Encontrados: } 3 \\
\text { Excluídos: } 3 \\
\text { Total: } 0\end{array}$ & $\begin{array}{l}\text { Encontrados: } 22 \\
\text { Excluídos: } 22 \\
\text { Total: } 0\end{array}$ \\
\hline
\end{tabular}

Fonte: Berty Ru Ping Song

\section{RESULTADOS}

O tamanho amostral variou de 16 a 1.596 participantes, totalizando 3.231 dentre os 13 estudos incluídos nesta revisão.

Em relação aos países de origem duas foram conduzidas no Brasil, duas na Irlanda, duas nos Estados Unidos, duas no Chile, duas na Espanha, uma na Holanda, uma na Nova Zelândia e por fim, uma na Costa Rica. O número de autores variou de 2 a 12.

Os artigos foram organizados com sua autoria/ano, amostra/tipo de estudo, metodologia, objetivos e conclusões, conforme Quadro 2.

Quadro 2. Caracterização dos estudos quanto à autoria/ano, amostra/tipo de estudo, metodologia, objetivos e conclusões

\begin{tabular}{|l|l|l|l|l|}
\hline Autoria/ & Amostra/Tip & Metodologia & $\begin{array}{l}\text { Objetivos/Foc } \\
\text { o do estudo }\end{array}$ & $\begin{array}{l}\text { Conclusões/Principa } \\
\text { is resultados }\end{array}$ \\
\hline Ano & o de Estudo & & do
\end{tabular}




\begin{tabular}{|c|c|c|c|c|}
\hline $\begin{array}{l}\text { Pierce M.; } \\
\text { Ramsey, } \\
\text { K.; Pinter, } \\
\text { J. } \\
\text { (2019) }\end{array}$ & $(n=412)$ & $\begin{array}{l}\text { Foi calculado as } \\
\text { taxas de } \\
\text { obesidade e } \\
\text { sobrepeso por } \\
\text { faixa etária e } \\
\text { examinado } \\
\text { possíveis } \\
\text { associações } \\
\text { com } \\
\text { comorbidades } \\
\text { comuns a partir } \\
\text { dos bancos de } \\
\text { dados. Também } \\
\text { foi examinado o } \\
\text { percentil médio } \\
\text { do índice de } \\
\text { massa corporal } \\
\text { (IMC) e a } \\
\text { mudança no } \\
\text { percentil do IMC } \\
\text { por idade. }\end{array}$ & $\begin{array}{l}\text { Avaliar } \\
\text { tendências } \\
\text { em } \\
\text { obesidade e } \\
\text { excesso de } \\
\text { peso em } \\
\text { crianças com } \\
\text { SD de } \\
\text { Oregon. }\end{array}$ & $\begin{array}{l}\text { Um total de } 23 \% \\
\text { estava com } \\
\text { sobrepeso e } 20,6 \% \\
\text { com obesidade. O } \\
\text { percentil do IMC } \\
\text { aumentou com o } \\
\text { sexo feminino, idade } \\
\text { e percentil de altura } \\
\text { para a idade. }\end{array}$ \\
\hline $\begin{array}{l}\text { Amo- } \\
\text { Setién; et } \\
\text { al. } \\
\text { (2019) }\end{array}$ & $\begin{array}{l}\text { Estudo } \\
\text { transversal }\end{array}$ & $\begin{array}{l}\text { Foi utilizado o } \\
\text { índice de massa } \\
\text { corporal, para } \\
\text { determinar o } \\
\text { estado } \\
\text { nutricional, } \\
\text { classificado em } \\
\text { quatro } \\
\text { categorias de } \\
\text { acordo com os }\end{array}$ & $\begin{array}{l}\text { Determinar a } \\
\text { prevalência } \\
\text { e fatores } \\
\text { associados } \\
\text { ao sobrepeso } \\
\text { e à obesidade } \\
\text { entre alunos } \\
\text { de escolas de } \\
\text { educação } \\
\text { especial. }\end{array}$ & $\begin{array}{l}\text { A prevalência de } \\
\text { sobrepeso/obesidad } \\
\text { e foi de } 40,9 \% \text {. A } \\
\text { obesidade foi mais } \\
\text { frequente no sexo } \\
\text { feminino }(26,0 \%) \text { do } \\
\text { que no masculino } \\
(9,8 \%) \text {. }\end{array}$ \\
\hline
\end{tabular}




\begin{tabular}{|c|c|c|c|c|}
\hline & & $\begin{array}{l}\text { critérios da } \\
\text { Força-Tarefa } \\
\text { Internacional } \\
\text { para a } \\
\text { Obesidade } \\
\text { (IOTF): baixo } \\
\text { peso; peso } \\
\text { normal; excesso } \\
\text { de peso; } \\
\text { obesidade. }\end{array}$ & & \\
\hline $\begin{array}{l}\text { O' Shea; } \\
\text { et al. } \\
(2018)\end{array}$ & $\begin{array}{l}\text { Estudo } \\
\text { transversal } \\
(n=61)\end{array}$ & $\begin{array}{l}\text { Mediu-se altura } \\
\text { e peso de } 61 \\
\text { participantes } \\
\text { com idades } \\
\text { entre } 4 \text { e } 16 \\
\text { anos. O índice } \\
\text { de massa } \\
\text { corporal (IMC) } \\
\text { foi calculado e o } \\
\text { percentual de } \\
\text { gordura corporal } \\
\text { (\%GC) foi } \\
\text { medido usando } \\
\text { a análise de } \\
\text { bioimpedância } \\
\text { elétrica (BIA). }\end{array}$ & $\begin{array}{l}\text { Determinar a } \\
\text { prevalência } \\
\text { de sobrepeso } \\
\text { e obesidade } \\
\text { entre } \\
\text { crianças e } \\
\text { jovens com } \\
\text { SD. }\end{array}$ & $\begin{array}{l}\text { A partir dos cálculos, } \\
51,6 \% \text { dos homens } \\
\text { e } 40 \% \text { das mulheres } \\
\text { estavam com } \\
\text { sobrepeso/obesidad } \\
\text { e em comparação } \\
\text { com } 32 \% \text { e } 14,8 \% \text {, } \\
\text { respectivamente, } \\
\text { usando \%GC. }\end{array}$ \\
\hline $\begin{array}{l}\text { De la } \\
\text { Piedra, M. } \\
\text { J.; et al. } \\
\text { (2017) }\end{array}$ & $\begin{array}{l}\text { Estudo } \\
\text { retrospectiv } \\
\text { o }\end{array}$ & $\begin{array}{l}\text { Foi avaliado o } \\
\text { perfil lipídico da } \\
\text { amostra, obtido } \\
\text { através dos } \\
\text { exames }\end{array}$ & $\begin{array}{l}\text { Descrever a } \\
\text { frequência de } \\
\text { dislipidemia } \\
\text { em uma } \\
\text { população de }\end{array}$ & $\begin{array}{l}\text { Após revisar os } \\
\text { prontuários, 58,3\% } \\
\text { apresentavam } \\
\text { algum tipo de } \\
\text { dislipidemia. }\end{array}$ \\
\hline
\end{tabular}




\begin{tabular}{|c|c|c|c|c|}
\hline & $(\mathrm{n}=218)$ & $\begin{array}{l}\text { laboratoriais. } \\
\text { Outras } \\
\text { condições foram } \\
\text { obtidas nos } \\
\text { prontuários. O } \\
\text { diagnóstico de } \\
\text { dislipidemia foi } \\
\text { considerado de } \\
\text { acordo com os } \\
\text { critérios } \\
\text { Instituto do } \\
\text { Nacional de } \\
\text { Coração, } \\
\text { Pulmões } \\
\text { Sangue } \\
\text { EUA 2011. }\end{array}$ & $\begin{array}{l}\text { crianças e } \\
\text { adolescentes } \\
\text { chilenos com } \\
\text { SD. }\end{array}$ & \\
\hline $\begin{array}{l}\text { Bertapelli, } \\
\text { F; et al. } \\
\text { (2016) }\end{array}$ & $\begin{array}{l}\text { Revisão da } \\
\text { literatura }\end{array}$ & $\begin{array}{l}\text { Foi realizada } \\
\text { uma busca } \\
\text { utilizando bases } \\
\text { de dados. De } \\
\text { um total de } \\
4.280 \text { estudos, } \\
\text { incluímos } 45 \\
\text { artigos de } \\
\text { pesquisa } \\
\text { originais } \\
\text { publicados entre } \\
1988 \text { e } 2015 \text {. }\end{array}$ & $\begin{array}{l}\text { Revisar a } \\
\text { prevalência } \\
\text { de sobrepeso } \\
\text { e obesidade e } \\
\text { seus } \\
\text { determinante } \\
\text { s em jovens } \\
\text { com SD. }\end{array}$ & $\begin{array}{l}\text { A prevalência } \\
\text { combinada de } \\
\text { sobrepeso } \\
\text { obesidade variou } \\
\text { entre os estudos de } \\
23 \% \text { a } 70 \% .\end{array}$ \\
\hline $\begin{array}{l}\text { Basil, J. } \\
\text { S.; et al. } \\
(2016)\end{array}$ & $\begin{array}{l}\text { Revisão } \\
\text { retrospectiv } \\
\text { a }\end{array}$ & $\begin{array}{l}\text { Avaliou-se } 303 \\
\text { crianças de } 2 \text { a } \\
18 \text { anos com }\end{array}$ & $\begin{array}{lr}\text { Avaliar } & \text { se } \\
\text { crianças } & \text { com } \\
\text { SD } & \text { nos }\end{array}$ & $\begin{array}{lr}\text { Dos } 303 \text { indivíduos } \\
\text { avaliados, } 47,8 \% \\
\text { eram } \quad \text { obesos, }\end{array}$ \\
\hline
\end{tabular}




\begin{tabular}{|c|c|c|c|c|}
\hline & $(n=303)$ & $\begin{array}{l}\text { diagnóstico de } \\
\text { SD. }\end{array}$ & $\begin{array}{l}\text { Estados } \\
\text { Unidos } \\
\text { apresentam } \\
\text { risco } \\
\text { aumentado } \\
\text { de } \\
\text { obesidade. }\end{array}$ & $\begin{array}{l}\text { significativamente } \\
\text { maior do que a } \\
\text { população } \\
\text { pediátrica geral, que } \\
\text { teve uma taxa de } \\
\text { obesidade de } 12,1 \%\end{array}$ \\
\hline $\begin{array}{l}\text { Hoey, } E \text {; } \\
\text { et al. } \\
(2016)\end{array}$ & $(n=131)$ & $\begin{array}{l}\text { Foram coletados } \\
\text { dados por meio } \\
\text { de } \\
\text { questionários; } \\
\text { inquérito } \\
\text { alimentar de } 4 \\
\text { dias e medidas } \\
\text { de peso, altura e } \\
\text { circunferência } \\
\text { da cintura. }\end{array}$ & $\begin{array}{l}\text { Examinar a } \\
\text { ingestão } \\
\text { nutricional e o } \\
\text { estado } \\
\text { antropométric } \\
\text { o de } \\
\text { indivíduos } \\
\text { com } \\
\text { deficiência } \\
\text { intelectual. }\end{array}$ & $\begin{array}{l}\text { O IMC médio dos } \\
\text { participantes foi de } \\
29,4 \mathrm{~kg} / \mathrm{m}^{2}+/-6,1 \text {, } \\
\text { onde } 28,2 \% \\
\text { estavam } \\
\text { sobrepeso e } 46,8 \% \\
\text { eram obesos. }\end{array}$ \\
\hline $\begin{array}{l}\text { Jiménez, } \\
\text { L.; et al. } \\
\text { (2015) }\end{array}$ & $\begin{array}{l}\text { Estudo de } \\
\text { corte } \\
\text { transversal, } \\
\text { descritivo } \\
(n=79)\end{array}$ & $\begin{array}{l}\text { Foram coletados } \\
\text { o peso altura e } \\
\text { também } \\
\text { calculado seu } \\
\text { IMC. } \\
\text { diagnóstico } \\
\text { nutricional foi } \\
\text { dado a partir do } \\
\text { IMC. }\end{array}$ & $\begin{array}{l}\text { Determinar o } \\
\text { excesso de } \\
\text { peso e taxas } \\
\text { de obesidade } \\
\text { em alunos } \\
\text { com SD. }\end{array}$ & $\begin{array}{l}\text { Taxas de sobrepeso } \\
\text { e obesidade de } \\
\text { acordo } \\
\text { SDM/2002, } \\
\text { NCHS/2000 } \\
\text { OMS/2007 foram } \\
43,57 \text { e } 66 \% \\
\text { respetivamente. }\end{array}$ \\
\hline $\begin{array}{l}\text { Bertapelli, } \\
\text { F.; et al. } \\
\text { (2013) }\end{array}$ & $(n=41)$ & $\begin{array}{l}\text { Foram } \\
\text { realizadas nove } \\
\text { medidas } \\
\text { antropométricas }\end{array}$ & $\begin{array}{l}\text { Avaliar a } \\
\text { prevalência } \\
\text { de obesidade } \\
\text { e topografia }\end{array}$ & $\begin{array}{l}\text { A maioria dos } \\
\text { indivíduos } \\
\text { apresentou excesso } \\
\text { de gordura corporal, }\end{array}$ \\
\hline
\end{tabular}




\begin{tabular}{|c|c|c|c|c|}
\hline & & $\begin{array}{l}\text {, a determinação } \\
\text { do \%GC e o } \\
\text { tratamento } \\
\text { estatístico. }\end{array}$ & $\begin{array}{l}\text { da gordura } \\
\text { corporal em } \\
\text { crianças e } \\
\text { adolescentes } \\
\text { de ambos os } \\
\text { sexos com } \\
\text { SD. }\end{array}$ & $\begin{array}{l}\text { sendo estes } \\
\text { superiores no sexo } \\
\text { feminino. } \\
\text { verificado também } \\
\text { maior concentração } \\
\text { de gordura na coxa } \\
\text { e menor depósito na } \\
\text { região do bíceps. }\end{array}$ \\
\hline $\begin{array}{l}\text { Van } \\
\text { Gameren- } \\
\text { Oosterom, } \\
\text { H.B. M.; et } \\
\text { al. } \\
(2012)\end{array}$ & $(n=1596)$ & $\begin{array}{l}\text { Foram coletados } \\
\text { os dados de } \\
\text { crescimento } \\
\text { longitudinal de } \\
\text { crianças } \\
\text { holandesas com } \\
\text { SD que } \\
\text { nasceram } \\
\text { depois de } 1982 .\end{array}$ & $\begin{array}{l}\text { Avaliar as } \\
\text { taxas de } \\
\text { prevalência } \\
\text { de sobrepeso } \\
\text { e obesidade } \\
\text { em uma } \\
\text { amostra } \\
\text { nacional de } \\
\text { crianças } \\
\text { holandesas } \\
\text { com SD. }\end{array}$ & $\begin{array}{l}\text { A avaliação mostrou } \\
\text { que } 25,5 \% \text { dos } \\
\text { meninos e } 32,0 \% \\
\text { das meninas } \\
\text { estavam acima do } \\
\text { peso, enquanto } \\
4,2 \% \text { dos meninos e } \\
5,1 \% \text { das meninas } \\
\text { estavam obesos. }\end{array}$ \\
\hline $\begin{array}{l}\text { Loveday, } \\
\text { S. J.; } \\
\text { Thompso } \\
\text { n, J.; } \\
\text { Mitchell, } \\
\text { E. A. } \\
\text { (2012) }\end{array}$ & $(n=77)$ & $\begin{array}{l}\text { Foram coletados } \\
\text { dados } \\
\text { antropométricos } \\
\text {, análise de } \\
\text { bioimpedância } \\
\text { elétrica (BIA) e } \\
\text { absorciometria } \\
\text { de raios-X de } \\
\text { dupla energia } \\
\text { (DXA) de } \\
\text { setenta crianças }\end{array}$ & $\begin{array}{lr}\text { Medir } & \text { com } \\
\text { precisão } & \text { a } \\
\text { gordura } & \\
\text { corporal } & \text { em } \\
\text { crianças } & \text { com } \\
\text { SD. } & \end{array}$ & $\begin{array}{l}\text { A média do } \% \text { GC foi } \\
\text { de } 30,5 \% \text { para } \\
\text { meninas e } 22,5 \% \\
\text { para meninos. Um } \\
\text { total de } 38 \% \text { das } \\
\text { meninas e } 23 \% \text { dos } \\
\text { meninos eram } \\
\text { obesos de acordo } \\
\text { com os critérios } \\
\text { internacionais. }\end{array}$ \\
\hline
\end{tabular}




\begin{tabular}{|c|c|c|c|c|}
\hline & & $\begin{array}{l}\text { com SD para } \\
\text { cálculo do \%GC. }\end{array}$ & & \\
\hline $\begin{array}{l}\text { Marín, A. } \\
\text { S.; } \\
\text { Graupera, } \\
\text { J. M. X. } \\
\text { (2011) }\end{array}$ & $(n=39)$ & $\begin{array}{l}\text { Foram } \\
\text { analisados a } \\
\text { composição } \\
\text { corporal, o nível } \\
\text { de atividade } \\
\text { física, ingestão } \\
\text { de nutrientes por } \\
\text { meio de } \\
\text { questionários } \\
\text { validados } \\
\text { parâmetros } \\
\text { bioquímicos } \\
\text { foram estimados } \\
\text { de } 38 \text { pessoas } \\
\text { com SD. }\end{array}$ & $\begin{array}{l}\text { Avaliar } \quad 0 \\
\text { estado } \\
\text { nutricional em } \\
\text { adultos } \\
\text { jovens com } \\
\text { SD. }\end{array}$ & $\begin{array}{l}\text { Ao classificar o IMC, } \\
36,8 \% \\
\text { apresentaram } \\
\text { sobrepeso (IMC: } 25 \text { - } \\
29,9 \mathrm{~kg} / \mathrm{m}^{2} \text { ) e } 36,8 \% \\
\text { eram obesos (IMC } \geq \\
\left.30 \mathrm{~kg} / \mathrm{m}^{2}\right) .\end{array}$ \\
\hline $\begin{array}{l}\text { Loría, A. } \\
\text { M.; } \\
\text { Urrutia, A. } \\
\text { R. G. } \\
\text { (2009) }\end{array}$ & $(n=16)$ & $\begin{array}{l}\text { Foram avaliados } \\
\text { indicadores } \\
\text { antropométricos } \\
\text {, bioquímicos e } \\
\text { clínicos. } \\
\text { Também foram } \\
\text { considerados } \\
\text { hábitos } \\
\text { alimentares, } \\
\text { estilo de vida e } \\
\text { aspectos } \\
\text { socioeconômico } \\
\text { s. }\end{array}$ & $\begin{array}{l}\text { Avaliar } 0 \\
\text { estado } \\
\text { nutricional de } \\
\text { crianças com } \\
\text { SD de } 7 \text { a } 14 \\
\text { anos. }\end{array}$ & $\begin{array}{l}\text { A maioria deles } \\
\text { apresentava sinais } \\
\text { de obesidade. Da } \\
\text { amostra avaliada } \\
80 \% \text { dos } \\
\text { participantes } \\
\text { apresentavam } \\
\text { hipertrigliceridemia } \\
\text { e } 73,33 \% \\
\text { apresentavam } \\
\text { níveis baixos de } \\
\text { colesterol HDL. } \\
\text { Além disso, foi } \\
\text { relatado um baixo }\end{array}$ \\
\hline
\end{tabular}


nível de atividade física.

\section{DISCUSSÃO}

\subsection{PREVALÊNCIA DE SOBREPESO E OBESIDADE}

A Síndrome de Down está significativamente associada a maiores taxas de sobrepeso e obesidade, com uma média de $26,70 \%$ e $32,98 \%$, respectivamente, em crianças e adolescentes de ambos os sexos. $O$ estado nutricional desta população pode ser determinado a partir do IMC, \%GC e BIA (WRIGHT et al., 2008).

Ao comparar com a população pediátrica geral, há uma taxa de aproximadamente duas vezes mais alta de sobrepeso e obesidade na população com SD (DE LA PIEDRA et al., 2017; BERTAPELLI et al., 2013). Esse quadro clínico facilita o desenvolvimento de outros problemas de saúde, como por exemplo, doenças cardiovasculares, dislipidemias, SAOS comprometendo a qualidade de vida dos indivíduos (LORÍA; URRUTIA, 2009; RIMMER et al., 2009).

Um estudo avaliou a má nutrição por excesso de peso em 79 estudantes com SD utilizando três padrões distintos de IMC para idade: SDM/2002, NCHS/2000 e OMS/2007. As taxas de obesidade encontradas foram de 21,5\%,34,2\% e 37,9\% para os padrões SDM/2002, NCHS/2000 e OMS/2007, respectivamente. Além disso, foi observado também que $60,8 \%$ dos estudantes avaliados possuíam alimentação inadequada, sendo esta um fator importante para explicar o excesso de peso (JIMÉNEZ et al., 2015).

Embora o período crítico para o desenvolvimento de sobrepeso e obesidade durante a infância e adolescência ainda não seja definido, é possível observar o aumento das taxas de tais condições a partir dos 2 anos de idade (BERTAPELLI et al., 2016). 


\subsection{CONSUMO ALIMENTAR E ATIVIDADE FÍSICA}

Uma alimentação é considerada saudável quando há um equilíbrio entre todos os nutrientes necessários, desde os macronutrientes (carboidratos, lipídios e proteínas) até os micronutrientes (vitaminas e minerais). Uma alimentação adequada é essencial para o bom funcionamento do organismo e para um crescimento e desenvolvimento saudável, além de prevenir o excesso de peso e outras comorbidades associadas. Sendo assim, ela é fundamental na melhora de qualidade de vida tanto para a população geral como também para a população com SD (VALLE, 2007).

Indivíduos com SD apresentaram o hábitos dietéticos não balanceados, sendo o consumo de energia, fibras, cálcio, vitamina $D$, folato e vitamina $E$ abaixo do recomendado. Ao contrário do caso dos açúcares, lipídios e gorduras saturadas, que há uma ingestão acima da quantidade recomendada por parte dessa população (HOEY et al., 2016; MARÍN; GRAUPERA, 2011).

Os alimentos mais consumidos são massas, pão com geleia ou mortadela ou manteiga, leite, suco industrializado, frutas mistas, bolachas, sobremesas, refrigerantes, farinhas e tubérculos. Observa-se que o consumo de carnes, frutas in natura, verduras e legumes é baixa o que significa um baixo consumo de fibras, fitoquímicos, vitaminas e minerais (LORIA; URRUTIA, 2009).

Além disso, os níveis de atividade física de para tal grupo pode ser considerado como sedentário, não é comum crianças e adolescentes praticarem exercícios físicos além das tarefas cotidianas (caminhar, correr pela casa, brincar, dançar) (BERTAPELLI et al., 2013; LORIA; URRUTIA, 2009).

O principal fator para manter um estado nutricional eutrófico é a alimentação adequada somado a pelo menos prática de atividade física leve ou moderada. Quando nenhum dos dois é realizado, os riscos de desenvolver sobrepeso e obesidade são aumentados e de forma síncrona os riscos de outras patologias também (HOEY et al., 2016; LORIA; URRUTIA, 2009; VALLE, 2007). 


\subsection{MEDIDAS ANTROPOMÉTRICAS}

De acordo com a classificação do IMC, valores entre 25 e $29,9 \mathrm{~kg} / \mathrm{m}^{2}$ apontam sobrepeso e a partir de $30 \mathrm{~kg} / \mathrm{m}^{2}$ obesidade (HOEY, 2017). O \%GC pode ser obtido a partir das dobras cutâneas ou BIA (BERTAPELLI et al., 2013; LOVEDAY; THOMPSON; MITCHELL, 2012). As dobras cutâneas mais utilizadas são triciptal (DCT), biciptal (DCB), subescapular (DCSE), supra-ilíaca (DCSI), abdominal (DCAB), coxa $(\mathrm{CX})$ e perna (PE). Utiliza-se também o somatório das 7 dobras $(\Sigma$ dobras) (BERTAPELLI et al., 2013).

Enquanto IMC e \%GC elevados aumentam o risco de obesidade, a circunferência da cintura determina o risco para complicações metabólicas (HOEY, 2017; MARÍN; GRAUPERA, 2011).

Para determinar a prevalência de sobrepeso e obesidade, 61 indivíduos entre 4 e 16 anos participaram de um estudo em que foi coletado suas alturas e pesos para o cálculo do IMC e a composição corporal utilizando a BIA. A classificação dos IMC mostrou que $35,5 \%$ dos meninos e $30,0 \%$ das meninas estavam com sobrepeso e $16,1 \%$ dos meninos e 10,0\% das meninas apresentavam obesidade. Já os resultados do \%GC mostraram que $20,0 \%$ dos meninos e $11,1 \%$ das meninas estavam com sobrepeso e $12,0 \%$ dos meninos e $3,70 \%$ das meninas estavam obesas (O'SHEA et al., 2018).

Em 2016, Hoey et al. verificaram que a média do IMC masculino era de $28,8 \mathrm{~kg} / \mathrm{m}^{2} \mathrm{e}$ o feminino de $30,2 \mathrm{~kg} / \mathrm{m}^{2}$. Categorizando os mesmos, observou-se que $2,4 \%$ da amostra ( $n=131$ ) estavam com baixo peso, $22,6 \%$ eutróficos, $28,2 \%$ sobrepeso e por fim $46,8 \%$ obesos (HOEY et al., 2016).

Sendo assim, é presumível que as medidas antropométricas sejam bons parâmetros para evidenciar a prevalência de sobrepeso e obesidade na população com SD (PIERCE; RAMSEY; PINTER, 2019; HOEY et al., 2016). 


\subsection{COMORBIDADES}

O excesso de gordura corporal está associado a várias comorbidades, tais como doenças cardiovasculares, dislipidemias, SAOS, diabetes, entre outros (DE LA PIEDRA et al., 2017). Logo, a população com SD requer cuidados redobrados pelas condições clínicas (DE LA PIEDRA et al., 2017; BERTAPELLI et al., 2013). Alguns possuem outras patologias como hipotireoidismo, que exerce influências que elevam ainda mais as taxas de sobrepeso e obesidade (VAN GAMEREN-OOSTEROM et al., 2012).

A quantidade de gordura corporal é proporcional aos níveis de colesterol total, LDL e triglicerídeos e inversamente proporcional aos níveis de HDL. É bastante comum observar que crianças e adolescentes com SD apresentam HDL abaixo dos valores de referência e simultaneamente altos níveis de triglicerídeos (DE LA PIEDRA et al., 2017).

A SAOS é a obstrução das vias aéreas durante o sono, seja de forma completa ou incompleta. Já sabendo da predisposição da população com SD para obesidade, é importante destacar que esta é um dos maiores fatores de risco para SAOS (PIERCE; RAMSEY; PINTER, 2019; BASIL et al., 2016).

Num estudo com 303 pacientes foi observado que 74,0\% tinham alguma obstrução, sendo a maioria $(38,9 \%)$ de maneira mais leve. Porém, ao se tratar de obesidade, o risco aumenta para obstruções moderadas e graves (BASIL et al., 2016).

Diante disso, conclui-se que o quadro de excesso de peso aumenta o risco de desenvolver outras manifestações clínicas e, juntos podem comprometer a saúde e qualidade de vida das crianças e adolescentes com SD.

\subsection{GÊNERO E IDADE}

Sabe-se que ambos os sexos são suscetíveis ao excesso de peso, que é o fator determinante para o risco de obesidade, porém, o grupo feminino apresentou taxas maiores para tal (AMO-SETIÉN et al., 2019; MARÍN; GRAUPERA, 2011). A 
prevalência de obesidade variou de $67,0 \%$ e 88,0\% para as faixas etárias de 6 a 12 anos e 13 a 19 anos, podendo ser justificado pela transição do período de infância para a adolescência (BERTAPELLI et al., 2013).

Apesar das taxas de sobrepeso e obesidade serem consideradas constantes até a adolescência, um estudo com 77 famílias observou que no sexo masculino houve um maior valor de \%GC antes dos 12 anos. Enquanto isso, o sexo feminino mostrou maiores valores de \%GC após os 12 anos (LOVEDAY; THOMPSON; MITCHELL, 2012).

Em vista disso, mais pesquisas são necessárias para definir se há de fato uma relação entre sobrepeso e obesidade e idade nas crianças e adolescentes com SD (VAN GAMEREN-OOSTEROM et al., 2012; LOVEDAY; THOMPSON; MITCHELL, 2012).

\section{CONSIDERAÇÕES FINAIS}

Com base nos dados levantados neste estudo, foi possível identificar uma alta prevalência de sobrepeso e obesidade em crianças e adolescentes com SD. Tal quadro acomete indivíduos com SD saudáveis e com comorbidades, de ambos os sexos independendo da idade e o grau de deficiência.

O principal fator para essa tendência de ganho de peso excessivo foi inconclusivo, pois as combinações de alterações metabólicas são distintas e individuais. Alguns determinantes prováveis são a taxa metabólica basal diminuída, níveis de atividade física baixos e padrões dietéticos desfavoráveis. O monitoramento precoce diminui o risco de desenvolver implicações médicas futuras.

A predisposição ao ganho de peso e os riscos de saúde associados, combinados com a vulnerabilidade desta população, exigem intervenções efetivas a fim de prevenir o agravamento das condições físicas e fisiológicas. Sendo assim a inclusão de padrões dietéticos saudáveis conciliados a prática de atividade física é essencial para a saúde e para uma maior qualidade de vida dessa população. 
Com tudo, a obesidade é um fator de risco para várias outras comorbidades que se acentua em indivíduos com SD podendo ser observada a partir da primeira infância. Planos de intervenções são fundamentais para melhorar o estado nutricional e recuperar a qualidade de vida.

\section{REFERÊNCIAS}

AMO-SETIÉN, F.; ABAJAS-BUSTILLO, R.; SARABIA-COBO; PARÁS-BRAVO, P.; LEAL COSTA, C., REDONDO-FIGUERO, C., BANDINI, L. (2019). Prevalence and factors associated with overweight and obesity among Spanish students attending special education schools. Journal of Applied Research in Intellectual Disabilities. 33. doi: 10.1111/jar.12679.

BASIL, JS; SANTORO, SL; MARTIN, LJ; HEALY, KW; CHINI, BA; SAAL, HM. Retrospective Study of Obesity in Children with Down Syndrome. J Pediatr. 2016 Jun;173:143-8. doi: 10.1016/j.jpeds.2016.02.046. Epub 2016 Mar 14. PMID: 26987801.

BERTAPELLI, F; PITETTI, K; AGIOVLASITIS, S; GUERRA-JUNIOR, G. Overweight and obesity in children and adolescents with Down syndrome-prevalence, determinants, consequences, and interventions: A literature review. Res Dev Disabil. 2016 Oct;57:181-92. doi: 10.1016/j.ridd.2016.06.018. Epub 2016 Jul 19. PMID: 27448331.

BERTAPELLI, Fabio et al . Prevalence of obesity and the body fat topography in children and teenagers with down syndrome. Rev. bras. crescimento desenvolv. hum., São Paulo, v. 23, n. 1, p. 65-70, 2013.

BULL, MJ; Committee on Genetics. Health supervision for children with Down syndrome. Pediatrics. 2011 Aug;128(2):393-406. doi: 10.1542/peds.2011-1605. Epub 2011 Jul 25. Erratum in: Pediatrics. 2011 Dec;128(6):1212. PMID: 21788214. 
CHAVES, Aclesia Lima; CAMPOS, Cíntia Kátia; NAVARRO, Antonio Coppi. Relação da síndrome de down com a obesidade. RBONE-Revista Brasileira de Obesidade, Nutrição e Emagrecimento, v. 2, n. 11, 2012.

HOEY, E; STAINES, A; WALSH, D; CORBY, D; BOWERS, K; BELTON, S; MEEGAN, S; MCVEIGH, T; MCKEON, M; TRÉPEL, D; GRIFFIN, P; SWEENEY, MR. An examination of the nutritional intake and anthropometric status of individuals with intellectual disabilities: Results from the SOPHIE study. J Intellect Disabil. 2017 Dec;21(4):346-365. doi: 10.1177/1744629516657946. Epub 2017 Feb 7. PMID: 27402617.

JIMENEZ, Lily et al . Malnutrición por exceso: alta frecuencia de sobrepeso y obesidad en escolares chilenos con síndrome de Down. Rev. méd. Chile, Santiago, v. 143, n. 4, p. 451-458, abr. 2015.

LOVEDAY, SJ; THOMPSON, JM; MITCHELL, EA. Bioelectrical impedance for measuring percentage body fat in young persons with Down syndrome: validation with dual-energy absorptiometry. Acta Paediatr. 2012 Nov;101(11):e491-5. doi: 10.1111/j.1651-2227.2012.02821.x. Epub 2012 Sep 5. PMID: 22897200.

MADRIGAL LORIA, Alejandra; GONZALEZ URRUTIA, Ana Rocío. Estado nutricional de niños con Síndrome Down del Centro Nacional de Educación Especial de Costa Rica. Rev. costarric. salud pública, San José , v. 18, n. 2, p. 72-78, Dec. 2009.

MOREIRA, Lília MA; EL-HANI, Charbel N; GUSMAO, Fábio AF. A síndrome de Down e sua patogênese: considerações sobre o determinismo genético. Rev. Bras. Psiquiatr., São Paulo, v. 22, n. 2, p. 96-99, June 2000.

MOURA, A. B.; MENDES, A.; PERI, A.; PASSONI, C. R. M. S. Aspectos nutricionais em portadores da Síndrome de Down. Cadernos da escola de saúde, Curitiba, p.1$11,2009$. 
O' SHEA, M; O' SHEA, C; GIBSON, L; LEO, J; CARTY, C. The prevalence of obesity in children and young people with Down syndrome. J Appl Res Intellect Disabil. 2018 Nov;31(6):1225-1229. doi: 10.1111/jar.12465. Epub 2018 May 15. PMID: 29761882.

PIEDRA, MJ DE LA,; ALBERTI G, CERDA J, CÁRDENAS A, PAUL MA, LIZAMA M. Alta frecuencia de dislipidemias en niños y adolescentes con Síndrome de Down. Rev. chil. pediatr. Santiago, v. 88, n. 5, p. 595-601, 2017.

PIERCE, M; RAMSEY, K; PINTER, J. Trends in Obesity and Overweight in Oregon Children With Down Syndrome. Glob Pediatr Health. 2019 Apr 2;6:2333794X19835640. doi: 10.1177/2333794X19835640. PMID: 31044152; PMCID: PMC6446252.

RIMMER, JH; YAMAKI, K; LOWRY, BM; WANG, E; VOGEL, LC. Obesity and obesityrelated secondary conditions in adolescents with intellectual/developmental disabilities. J Intellect Disabil Res. 2010 Sep;54(9):787-94. doi: 10.1111/j.13652788.2010.01305.x. Epub 2010 Jul 12. PMID: 20630017.

SOLER MARIN, A.; XANDRI GRAUPERA, J. Mํ. Nutritional status of intellectual disabled persons with Down syndrome. Nutr. Hosp., Madrid , v. 26, n. 5, p. 10591066, oct. 2011.

SOUZA, Marcela Tavares de; SILVA, Michelly Dias da; CARVALHO, Rachel de. Revisão integrativa: o que é e como fazer. Einstein (São Paulo), São Paulo , v. 8, n. 1, p. 102-106, Mar. 2010.

THEODORO, Luciana Rodrigues; BLASCOVI-ASSIS, Silvana Maria. Síndrome de Down: associação de fatores clínicos e alimentares em adolescentes com sobrepeso e obesidade. Psicol. teor. prat., São Paulo, v. 11, n.1, p. 189-194, jun. 2009.

VALLE, Janaína Mello Nasser. A formação dos hábitos alimentares na infância: uma revisão de alguns aspectos abordados na literatura nos últimos dez anos. Aps, Juiz de Fora, v. 1, n. 10, p.56-65, jun. 2007. 
VAN Gameren-Oosterom, HB; van Dommelen, P; Schönbeck, Y; Oudesluys-Murphy, AM; van Wouwe, JP; Buitendijk, SE. Prevalence of overweight in Dutch children with Down syndrome. Pediatrics. 2012 Dec; 130(6):e1520-6. doi: 10.1542/peds.20120886. Epub 2012 Nov 12. PMID: 23147968.

WORLD HEALTH ORGANIZATION. Obesity: preventing and managing the global epidemic. Report of a WHO consultation on obesity in Geneva, 3-5, jun. 1997. Geneva: WHO, 1998.

WRIGHT, C.; SHERRIFF, A.; WARD, S. et al. Development of bioelectrical impedance-derived indices of fat and fat-free mass for assessment of nutritional status in childhood. Eur J Clin Nutr. 62, 210-217 https://doi.org/10.1038/sj.ejcn.1602714

Enviado: Novembro, 2020.

Aprovado: Novembro, 2020. 